\title{
The Effects of $17 \beta$ Estradiol, $17 \alpha$ Estradiol and Progesterone on Oxidative Stress Biomarkers in Ovariectomized Female Rat Brain Subjected to Global Cerebral Ischemia
}

\author{
V. H. OZACMAK, H. SAYAN \\ Zonguldak Karaelmas University, Medical School, Department of Physiology, Kozlu, Zonguldak, \\ Turkey
}

Received July 14, 2008

Accepted December 8, 2008

On-line December 17, 2008

\begin{abstract}
Summary
Neuroprotective effects of estrogens and progesterone have been widely studied in various experimental models. The present study was designed to compare possible neuroprotective effects of 17alpha-estradiol, 17beta-estradiol, and progesterone on oxidative stress in rats subjected to global cerebral ischemia. Global cerebral ischemia was induced in ovariectomized female rats by four vessel occlusion for $10 \mathrm{~min}$. Following $72 \mathrm{~h}$ of reperfusion, levels of malondialdehyde (MDA, oxidative stress marker), and reduced glutathione (GSH, major endogenous antioxidant) were assessed in hippocampus, striatum and cortex of rats treated with either 17alpha-estradiol, 17beta-estradiol, progesterone or estradiol + progesterone beforehand. Steroid administration ameliorated ischemia-induced decrease in GSH and increase in MDA levels. Our data offers additional evidence that estrogens and progesterone or combination of two exert a remarkable neuroprotective effect reducing oxidative stress.
\end{abstract}

\section{Key words}

Estradiol • Global cerebral ischemia - Progesterone • Oxidative stress

\section{Corresponding author}

H. Sayan, Zonguldak Karaelmas University, Medical School, Department of Physiology, 67600, Kozlu, Zonguldak, Turkey. Fax: +90 37226102 64. E-mail: halesayan@yahoo.com

It has been demonstrated in in vivo and in vitro studies that estrogen and progesterone are neuroprotective in models of acute neuronal stress and neurodegeneration (Amantea et al. 2005). Several mechanisms are conceivable for neuroprotection by estrogen: a genomic estrogen receptor-mediated pathway that involves gene transcription, a non-genomic signaling pathway associated with activation of signaling molecules such as mitogen-activated protein kinase and/or phosphatidylinositol-3-kinase/protein kinase B, and an antioxidant free-radical scavenging pathway (Garcia-Segura et al. 2001). One important protective mechanism of estradiol is through its antioxidant action. Estrogens suppress lipid peroxidation induced by amyloid $\beta$-peptide and ferrous sulfate (Kii et al. 2005) and attenuate cell death caused by oxidative stress in hippocampal cell line (Vedder et al. 1999). The mechanism for the antioxidative action of the estrogen is proposed to reduce the elevation of intracellular $\mathrm{Ca}^{2+}$ concentration, which is a major element in the development of ischemic damage by reactive oxygen species (Behl et al. 1995). Several members of the estrogen family are potent antioxidants with their phenolic structure in the steroid A ring being responsible for the inhibition of iron-catalyzed lipid peroxidation (Kii et al. 2005).

Progesterone also possesses neuroprotective properties following cerebral ischemia as well as traumatic brain injury (Roof and Hall 2000, Morali et al. 2005). Progesterone may reduce oxidative stress by its membrane stabilizing effect as well (Roof and Hall 2000).

A number of studies have investigated the effect of $17 \beta$ estradiol and progesterone or combined treatment 
Table 1. The effect of gonadal steroids on MDA content of cortex, hippocampus, and striatum in ovariectomized rats subjected to global cerebral ischemia.

\begin{tabular}{lccccccc}
\hline \multirow{2}{*}{ Groups } & \multicolumn{3}{c}{ MDA nmol/g } & & \multicolumn{3}{c}{ GSH $\boldsymbol{\mu m o l} / \mathbf{g}$} \\
\cline { 2 - 3 } \cline { 7 - 8 } & Striatum & Hippocampus & Cortex & & Striatum & Hippocampus & Cortex \\
\hline Sham Control & $166 \pm 34$ & $157 \pm 19$ & $153 \pm 21$ & & $2.41 \pm 0.18$ & $2.27 \pm 0.1$ & $1.35 \pm 0.09$ \\
$I / R$ Control & $351 \pm 29^{*}$ & $248 \pm 13^{*}$ & $313 \pm 20^{*}$ & & $1.45 \pm 0.15^{*}$ & $1.66 \pm 0.09^{*}$ & $0.51 \pm 0.06^{*}$ \\
$I / R+17 \alpha$ estradiol & $248 \pm 15$ & $206 \pm 18$ & $201 \pm 8^{\#}$ & & $2.32 \pm 0.04^{\#}$ & $2.51 \pm 0.2^{\#}$ & $0.67 \pm 0.07^{*}$ \\
$I / R+17 \beta$ estradiol & $207 \pm 3^{\#}$ & $175 \pm 15^{\#}$ & $168 \pm 5^{\#}$ & & $1.74 \pm 0.11$ & $2.3 \pm 0.1^{\#}$ & $1.29 \pm 0.12^{\#}$ \\
$I / R+$ Progesterone & $178 \pm 13^{\#}$ & $133 \pm 5^{\#}$ & $147 \pm 9^{\#}$ & & $2.19 \pm 0.04^{\#}$ & $2.25 \pm 0.16^{\#}$ & $0.88 \pm 0.08^{\#}$ \\
$I / R+17 \beta e s t r a d i o l+$ & $176 \pm 36^{\#}$ & $156 \pm 23^{\#}$ & $143.24^{\#}$ & & $1.61 \pm 0.22$ & $2.38 \pm 0.03^{\#}$ & $1.46 \pm 0.17^{*}$ \\
progesterone & & & & & & \\
\hline
\end{tabular}

Data are expressed as mean \pm S.E.M. $(n=6) . * P<0.05$ vs. sham control, \# $\mathrm{P}<0.05$ vs. I/R control.

(Murphy et al. 2002, Toung et al. 2004) against I/R injury. Since estrogen and progesterone have antioxidant potencies, we aimed to test hypothesis that gonadal steroids reduce ischemic neuronal damage by lowering oxidative stress, which is associated with decreased lipid peroxidation.

Thirty six adult female Wistar rats (4-6 months old, 200-250 g) were used. Animals were bilaterally ovariectomized under sodium thiopenthal anesthesia $(60 \mathrm{mg} / \mathrm{kg}$, i.p.) to eliminate endogenous estradiol and progesterone production. Ovariectomies were performed at 4-6 weeks prior to the study. All protocols followed in the study were approved by the Animal Care and Use Committee at the Zonguldak Karaelmas University Medical School.

Cerebral ischemia was generated by the method of four vessel occlusion for $10 \mathrm{~min}$. Six groups were designed: 1) sham-operated control group $(n=6)$, subjected to all surgical procedures except occlusion; 2) I/R control group treated with vehicle $(n=6) ; 3)$ I/R group treated with $17 \alpha$ estradiol $(n=6) ; 4)$ I/R group treated with $17 \beta$ estradiol $(n=6) ; 5) \quad \mathrm{I} / \mathrm{R}$ group treated with progesterone $(n=6) ; 6) \mathrm{I} / \mathrm{R}$ group treated with $17 \beta$ estradiol + progesterone $(n=6)$. The treatment paradigm was based on previously published studies (Santizo et al. 2000). Estradiol (0.1 $\mathrm{mg} / \mathrm{kg}$ i.p.) and progesterone (10 mg/kg i.p.), dissolved in DMSO, were given daily for one week preceding the study, whereas control animals were given vehicle only. Additional doses of drugs or vehicle were administered at 24, 48, and $72 \mathrm{~h}$ of reperfusion. Animals were sacrificed at the end of $72 \mathrm{~h}$ reperfusion period; brains were quickly removed and careful dissection of hippocampus, striatum, and cortex was performed in ice-cold saline. MDA was measured using a method described by Casini et al. (1986). Based on modified Ellman method, GSH content of the brain tissue samples was also measured (Aykaç et al. 1985).

$\mathrm{I} / \mathrm{R}$ caused significant increase in the MDA content of the tissue samples from cortex, hippocampus, and striatum (Table 1). Administration of steroids except $17 \alpha$ estradiol provided remarkable attenuation of the elevated MDA content in striatum and hippocampus, bringing them back to control levels. Among groups treated with steroids were no significant differences. In cortex, however, the treatment with all steroids was effective in reducing MDA level.

The treatment with steroids ameliorated I/R-induced decrease in GSH levels in hippocampus (Table 1). In cortex, GSH content of sham control group was not significantly different from that of $17 \beta$ estradiol group. I/R treated with $17 \alpha$ estradiol was not significantly different from I/R control in terms of GSH level, while the combined group was significantly different not only from I/R control but also from sham control. In striatum, only $17 \alpha$ estradiol- and progesterone-treated groups were significantly different from I/R control.

We observed that gonadal steroids appeared to show regional effect on lipid peroxidation and GSH homeostasis in rat brain. For instance, MDA levels of hippocampus and striatum decreased significantly in all groups except for $17 \alpha$ estradiol-treated animals, relative to $\mathrm{I} / \mathrm{R}$ group. Yet, MDA levels in cortex were lowered in all treatment groups. Acute or chronic treatment with $17 \beta$ estradiol in cerebral ischemia model has been shown to be protective. Moreover, the protection has also been observed in animals given the purportedly receptor inactive $17 \alpha$ estradiol. Although investigated only in a few studies, $17 \alpha$ estradiol attenuates brain lipid 
peroxidation and increases the survival promoting protein kinases (McClean and Nunez 2008). We observed in hippocampus and striatum that $17 \alpha$ estradiol was not as effective in reducing lipid peroxidation as $17 \beta$ estradiol. These findings appear to be consistent with a nongenomic process and might be considered as evidence for antioxidant action of estrogens, as has been suggested (Vedder et al. 1999). On the other hand, estrogen related neuroprotection, to some degree, may also involve interactions with classic receptors (Singer et al. 1996). Furthermore, the discrepancy in cortex in respect to two stereoisomers of estradiol may be explained by variations in regional responses of rat brain. Nevertheless, further studies are required to reveal more clearly how estradiol mediates the neuroprotection in brain.

We showed that the combined treatment reduced lipid peroxidation in all brain tissues. The mechanism by which progesterone provides protection from ischemic brain injury may be through its free radical scavenger action. Our finding is consistent with other reports in which progesterone alone protects hippocampal neurons against different forms of experimental brain injury (Ciriza et al. 2006). However, we observed no additive effects on reducing MDA levels and GSH metabolism in the combined treatment group. This is consistent with previous experiments showing that a combination of estrogen and progesterone has no additive effect on reducing ischemic brain injury (Toung et al. 2004). An explanation may be related to the fact that progesterone could modify the impact of estradiol in hippocampus (Galea et al. 2006).

GSH is an endogenous antioxidant scavenging free radicals and protecting against oxidative stress. Preserving GSH-mediated antioxidant defense is critical for cell survival (Anderson et al. 2004). GSH content was significantly increased in hippocampus of all the treatment groups. In cortex, treatments with $17 \beta$ estradiol and $17 \beta$ estradiol + progesterone alleviated I/R-induced decrease in GSH, whereas, in striatum, $17 \alpha$ estradiol or progesterone administration were effective to protect GSH levels. It is well known that sex steroid hormones modulate the enzyme activities in the antioxidant defense system (Pajovic et al. 2003). In the present study, $17 \beta$ estradiol, its non-estrogenic stereoisomer $17 \alpha$ estradiol, and progesterone protect GSH depletion in hippocampus, suggesting a non-receptor mechanism. However, $17 \alpha$ estradiol failed to preserve GSH content in cortex. This might be explained by $17 \alpha$ estradiol-induced consumption of GSH in cortical tissues. However, this consumption may not be effective in reducing lipid peroxidation.

Data of the present study provided an additional evidence for a possibility that the neuroprotective effects of estradiol and progesterone are mediated, at least in part, through reduction of oxidative stress and restoration of GSH levels after transient global cerebral ischemia. The beneficial effects of estrogens in the brain including the inhibition of lipid peroxidation may even have therapeutic consequences, as suggested by recent studies on the clinical potential of estrogens in neurodegenerative diseases (Simpkins and Dykens 2008).

\section{Conflict of Interest}

There is no conflict of interest.

\section{Acknowledgements}

The study was supported by Zonguldak Karaelmas University Research Project grant (\#2003-00-03).

\section{References}

AMANTEA D, RUSSO R, BAGETTA G, CORASANITI MT: From clinical evidence to molecular mechanisms underlying neuroprotection afforded by estrogens. Pharmacol Res 52: 119-132, 2005.

ANDERSON MF, NILSSON M, ERIKSSON PS, SIMS NR: Glutathione monoethyl ester provides neuroprotection in a rat model of stroke. Neurosci Lett 354: 163-165, 2004.

AYKAC G, UYSAL M, YALAN AS, KOCAK TOKER N, SIVAS A, OZ H: The effects of chronic ethanol injection on hepatic lipid peroxide, glutathione, glutathione peroxidase and glutathione transferase in rats. Toxicology 36: 71-76, 1985.

BEHL C, WIDMANN M, TRAPP T, HOLSBOER F: 17beta-Estradiol protects neurons from oxidative stress-induced cell death in vitro. Biochem Biophys Res Commun 216: 473-482, 1995.

CASINI A, FERRALI A, POMPELLA A, MAELLARO E, COMSARTI M: Lipid peroxidation and cellular damage in extrahepatic tissues of bromobenzene in toxicated mice. Ann J Pathol 123: 520-531, 1986. 
CIRIZA I, CARRERO P, FRYE CA, GARCIA-SEGURA LM: Reduced metabolites mediate neuroprotective effects of progesterone in the adult rat hippocampus. The synthetic progestin medroxyprogesterone acetate (Provera) is not neuroprotective. J Neurobiol 66: 916-928, 2006.

GALEA LA, SPRITZER MD, BARKER JM, PAWLUSKI JL: Gonadal hormone modulation of hippocampal neurogenesis in the adult. Hippocampus 16: 225-232, 2006.

GARCIA-SEGURA LM, AZCOITIA I, DONCARLOS LL: Neuroprotection by estradiol. Prog Neurobiol 63: 29-60, 2001.

KII N, ADACHI N, LIU K, ARAI T: Acute effects of 17 beta-estradiol on oxidative stress in ischemic rat striatum. J Neurosurg Anesthesiol 17: 27-32, 2005.

MCCLEAN J, NUÑEZ JL: 17alpha-Estradiol is neuroprotective in male and female rats in a model of early brain injury. Exp Neurol 210: 41-50, 2008.

MORALI G, LETECHIPIA-VALLEJO G, LOPEZ-LOEZA E, MONTES P, HERNANDEZ-MORALES L, CERVANTES M: Post-ischemic administration of progesterone in exerts neuroprotective effects on the hippocampus. Neurosci Lett 382: 286-290, 2005.

MURPHY SJ, LITTLETON-KEARNEY MT, HURN PD: Progesterone administration during reperfusion, but not preischemia alone, reduces injury in ovariectomized rats. J Cereb Blood Flow Metab 22: 1181-1188, 2002.

PAJOVIC SB, SAICIC ZS, SPASIC MB, PETROVIC VM: The effect of ovarian hormones on antioxidant enzyme activities in the brain of male rats. Physiol Res 52: 189-194, 2003.

ROOF RL, HALL ED: Gender differences in acute CNS trauma and stroke: neuroprotective effects of estrogen and progesterone. J Neurotrauma 17: 367-388, 2000.

SANTIZO RA, ANDERSON S, YE S, KOENIG HM, PELLIGRINO DA: Effects of estrogen on leukocyte adhesion after transient forebrain ischemia. Stroke 31: 2231-2235, 2000.

SIMPKINS JW, DYKENS JA: Mitochondrial mechanisms of estrogen neuroprotection. Brain Res Rev 57: 421-430, 2008.

SINGER CA, ROGERS KL, STRICKLAND TM, DORSA DM: Estrogen protects primary cortical neurons from glutamate toxicity. Neurosci Lett 212: 13-16, 1996.

TOUNG TJ, CHEN TY, LITTLETON-KEARNEY MT, HURN PD, MURPHY SJ: Effects of combined estrogen and progesterone on brain infarction in reproductively senescent female rats. J Cereb Blood Flow Metab 24: 1160$1166,2004$.

VEDDER H, ANTHES N, STUMM G, WÜRZ C, BEHL C, KRIEG JC: Estrogen hormones reduce lipid peroxidation in cells and tissues of the central nervous system. J Neurochem 72: 2531-2538, 1999. 